\title{
Statistical analysis of the inherent variability in the results of evolutionary debris models
}

\author{
Aleksander A. Lidtke ${ }^{1,}$ \\ University of Southampton, Southampton, SO17 1BJ, UK \\ Hugh G. Lewis ${ }^{2}$, \\ University of Southampton, Southampton, SO17 1BJ, UK \\ Roberto Armellin ${ }^{3}$, \\ Surrey Space Centre, University Of Surrey, Guildford, GU2 7XH, UK
}

\begin{abstract}
Space debris simulations, e.g. those performed by the Inter-Agency Debris Coordination Committee (Liou et al., 2013), showed that the number of objects in orbit is likely to increase. This study analyses the uncertainty in the results of space debris simulations performed using semi-stochastic models that necessitate the use of Monte Carlo simulations, which are often used by the Inter-Agency Debris Coordination Committee, amongst other studies. Statistics of the possible numbers of objects in orbit and collisions over the next 200 years are generated for the "mitigation only" scenario using a sample of 25000 Monte Carlo runs. Bootstraps on the mean, median, variance, skewness and kurtosis of these distributions are performed. It is shown that the distribution of the objects predicted to be on-orbit becomes log-normal as collisions occur, and that Monte Carlo samples larger than traditionally used are needed to capture the debris simulation uncertainty.
\end{abstract}

Keywords: orbital debris, modelling, Monte Carlo, uncertainty, bootstrap

Email addresses: al11g09@soton.ac.uk (Aleksander A. Lidtke),

H.G.Lewis@soton.ac.uk r.armellin@surrey.ac.uk (Roberto Armellin)

${ }^{1} \mathrm{PhD}$ student, Astronautics Research Group, University of Southampton, UK

${ }^{2}$ Senior Lecturer, Astronautics Research Group, University of Southampton, UK

${ }^{3}$ Senior Lecturer, Surrey Space Centre, University Of Surrey, Guildford, UK 


\section{Introduction}

The satellite launches that have taken place to date have led to a build-up of objects in Earth orbit. The number of derelict objects exceeded that of operational satellites in the early nineteen-sixties (Johnson, 2005). Approximately a decade later it was realised for the first time that the space debris would accumulate in Earth orbits and potentially create more debris through mutual collisions (Kessler \& Cour-Palais, 1978). By early March 2016, the catalogue of space objects, which can be tracked and whose positions were made publicly available through Space-Track (2016), had grown to 15447 .

Kessler \& Cour-Palais (1978) proposed a situation where accumulation of objects in orbit would trigger collisions creating new debris that would lead to more collisions. Such a "collision cascade" is often referred to as the "Kessler syndrome". Since this early work, space debris modelling has been extended to understand how the space debris environment is likely to evolve and what actions may be needed to ensure the safety of spacecraft operations in the future. In particular, Liou \& Johnson (2008) suggested, based on the results of LEGEND (LEO-to-GEO environment debris model), that the number of objects in orbit might keep increasing even if no future launches take place.

The authors of an Inter-Agency Debris Coordination Committee (IADC) study (Liou et al., 2013) suggested that aggressive measures should be considered to stabilise the low-Earth orbit (LEO) environment (Liou, 2011; Furuta et al., 2014). Removal of large, derelict objects, i.e. active debris removal (ADR), has been suggested by several authors (Liou \& Johnson, 2009; Braun et al., 2013; McKnight et al., 2014; Furuta et al., 2014; Wormnes et al., 2013; Pas et al., 2014) as a means to prevent the growth of the number of objects in the debris environment. If five derelicts that are large in mass and have the highest, relative to other objects, probability of collision are removed every year, beginning in 2020, the total number of objects on-orbit is predicted to be approximately constant over the next 200 years (Liou \& Johnson, 2009). However, the assumptions of the debris models give rise to uncertainties in the prediction results, which might affect decision-making related to actions needed to preserve the space environment.

This paper briefly reviews certain space debris modelling approaches that have been used to date, notably the ones that formed a part of the IADC 
comparison study (Liou et al., 2013). The variability in the results inherent to this subset of the current debris models, which use a Monte Carlo (MC) approach to generate statistics of the future debris environment, is highlighted and its origins identified. An exemplar scenario was simulated using the Debris Analysis and Monitoring Architecture to the Geosynchronous Environment (DAMAGE), the evolutionary debris model developed at the University of Southampton (Lewis et al., 2001). The relationship between the shape of the distributions of the number of objects in the Monte Carlo sample and the number of $\mathrm{MC}$ runs in this sample is investigated at four census epochs. The same analysis is performed for the number of collisions that occurred in every $\mathrm{MC}$ run during the projection, and conclusions are drawn.

\section{Space debris modelling approaches}

One of the first space debris models, devised by Kessler \& Cour-Palais (1978) and based on the work by Oepik (1952), used the kinetic theory of gases to compute the average number of collisions per unit time for the objects in the debris environment, i.e. the collision rates (Kessler, 1981). Possibly the most significant assumption made by Kessler (1981) is that the volume, where the collision probabilities between objects are computed, is small compared to the uncertainties on the orbits of the objects. In an extreme case, if this volume is large enough to include all Earth-orbiting objects, this assumption means that every satellite could collide with any other. Even if this volume is smaller, it is impossible to predict which objects are going to be involved in collisions, because the objects could be located anywhere in the small volumes of space. Therefore, random numbers are generated and compared to the collision rates to decide which objects collide in the simulations (Liou \& Johnson, 2006). The use of random numbers, which are also employed to simulate explosions, on-orbit failures etc. (Liou \& Johnson, 2008), gives rise to the fact that the debris models predict a range of possible evolutions of the number of objects. Hence, these models can be referred to as semi-stochastic, i.e. some of their elements are stochastic, e.g. occurrence of collisions, but others deterministic e.g. solar activity predictions. However, the use of the random numbers to simulate collisions stems from the fact that the kinetic theory of gases cannot predict the actual collisions.

Predicting the actual close approaches between objects, and estimating the 
associated collision probabilities based on the uncertainties of their states at the times of the closest approaches, is impossible on time scales of hundreds of years. This is because the positions of the objects are subject to orbit determination uncertainties, which grow during propagation (Vallado \& Seago, 2009) under the influence of orbital dynamics. Therefore, modelling the evolution of the space debris on such long time scales cannot rely on actual collision forecasts. Instead, collision rates, computed using approaches based on the work by Kessler \& Cour-Palais (1978), are used. Specifically, two different types of derived debris models are being used today: particles-in-box (PIB) and three-dimensional semi-stochastic approaches. These models have been in the development since the 1960s, and the dates of the corresponding references given herein give more up-to-date descriptions of the used algorithms. The purpose of the remainder of this section is not to review the history of the debris modelling but to give the Reader an overview of how the models function.

The PIB models, for example by Farinella \& Cordelli (1991), Talent (1992), Kebschull et al. (2014) or Lewis et al. (2009), describe the evolution of the number of objects on-orbit $(N)$ with differential equations. This set of equations expresses the rate of change of $N$ as a function with coefficients corresponding to deposition $(A)$, decay $(B)$, and collisions $(C)$ of objects (Talent, 1992):

$$
\frac{d N}{d t}=A+B N+C N^{2}
$$

Integrating Eq. 1, starting at some initial conditions, yields the number of objects in orbit at the end of the integration period. Fundamentally, the kinetic theory of gases manifests itself in the $C N^{2}$ term, which states that any object can collide with any other. These models were further improved by, for example, only allowing objects from distinct altitude bins to collide with each other (Kebschull et al., 2014). However, the assumption of the kinetic theory of gases has been retained.

The other group of models, i.e. the three-dimensional semi-stochastic codes, also uses the kinetic theory of gases assumption, however at a different spatial scale. These models typically examine every object separately and compute the collision probability for the examined object (target) using the flux of other objects at that target (Klinkrad, 2006; Bastida-Virgili, 2016; Liou, 2006). This flux $F$, in $m^{-2} s^{-1}$ (or other units of length and time), is computed by assuming uniform probability of residence of two objects in 
small volumes of space, which uses the kinetic theory of gases assumption in these volumes (Klinkrad, 2006). Different shapes of these volumes are used, e.g. Liou (2006) and Lewis et al. (2001) use cubes, Ariyoshi \& Hanada (2009) spheres, and Bastida-Virgili (2016) bins in right ascension-declinationaltitude space. The volumes of space are small relative to the PIB models Liou (2006) recommends using cubes with edge length less than $1 \%$ of the average semi-major axis of all the objects (specifically $10 \mathrm{~km}$ ). However, if the volumes were increased to include all the Earth orbiting objects, the particles-in-box model would be recovered (Liou, 2006). When the flux $F$ is known, the collision probability $P_{C}$ is computed as $P_{C}=F \sigma \Delta t$, where $\sigma$ is the combined cross-sectional area of the objects and $\Delta t$ is the time step (Klinkrad, 2006). To get accurate representation of $P_{C}$ at any point in the simulation time, $\Delta t$ should be equal to the time the objects spend in the small volume of space. However, the smaller the $\Delta t$, the more computational time the simulation requires. Thus, due to computational time restrictions, larger time steps are normally used.

A random number is generated for every conjunction and a collision simulated if the random number is lower than $P_{C}$ (Liou \& Johnson, 2006). The number of fragments resulting from every collision is then computed using the NASA Standard Breakup Model (Johnson et al., 2001) or a similar empirical algorithm. The breakup model introduces randomness into the simulations through the use of random samples drawn from probability distributions describing fragment characteristics.

In this study, DAMAGE was used. Collisions in every Monte Carlo run of DAMAGE, and the corresponding numbers of fragments, are generated based on random numbers, hence every $\mathrm{MC}$ runs is unique. Moreover, close approaches, for which $P_{C}$ is computed, are found by randomising mean anomaly of all the objects and identifying pairs of objects that are within a certain distance threshold from one another while their mean anomaly is being randomised. This introduces further source of variability between different MC runs.

Currently, the semi-stochastic evolutionary debris models are predominantly used for international, collaborative studies. Most importantly, only such models were used in the IADC comparison study (Liou et al., 2013). These simulation tools are used to probe plausible future scenarios and enable additional data, e.g the relative effectiveness of various mitigation measures, to be extracted from the simulations (DoladoPerez et al., 2015; Lewis et al., 2009). The use of random numbers creates a need for large Monte Carlo 
samples that have to be generated in order to establish statistics about the expected number of collisions, their severities and the total numbers of objects in orbit (Liou \& Johnson, 2006). Liou \& Johnson (2008) suggested that 10 to $40 \mathrm{MC}$ runs are sufficient for this purpose.

Multiple sources of uncertainty, both dependent and independent of the debris model implementation details, affect the results of such models. These factors are reviewed by DoladoPerez et al. (2015) and influence the number of objects in orbit and how well this can be predicted. The uncertain, modelindependent parameters, e.g. the solar activity or the future launch traffic, can be included in the Monte Carlo analyses as done by e.g. White \& Lewis (2014). From the uncertainty sources, which depend on the assumptions of the evolutionary debris model in use, orbital collisions have been found to be the source of the largest variability between different Monte Carlo runs (Liou \& Johnson, 2008; Lewis et al., 2009). Both sets of uncertain parameters mean that the predictions of the future debris environment generate a distribution of the possible number of objects in orbit. However, if sufficiently many $M C$ runs are analysed, these distributions can be established. Research questions can be answered based on those, for example the relative effectiveness of various debris mitigation or remediation measures can be compared and decisions whether to implement any can be taken.

This paper will not investigate the uncertainty in the future prediction of the number of objects in orbit that arises due to factors independent of the debris models. Instead, it will focus on the inherent variability in the data generated by DAMAGE. The algorithms used to simulate collisions implemented in DAMAGE are similar to those used by LEGEND and SDM (Space Debris Mitigation), but their specifics are different from LEODEEM (LEO Space Debris Environment Evolution Model), LUCA (Long Term Utility for Collision Analysis) and DELTA (Debris Environment Long-Term Analysis), for example (Liou et al., 2013). Even though the details of all the models are different, the number of objects on orbit in 2209 predicted by them agrees to within $10 \%$ (error relative to the mean of all the models) (Liou et al., 2013). What is more, collision rate calculation in LUCA is also based on the work by Oepik (1952) (Radtke et al., 2013), and DELTA also uses flux of the objects to compute the $P_{C}$ (Bastida-Virgili, 2016). Thus, it is believed that analysing the variability inherent to DAMAGE is important to understand the conclusions that can be drawn using other semi-stochastic debris models, specifically the ones used by the IADC.

The mechanism that leads to large variability between the Monte Carlo 
runs, even if no uncertainty on e.g. the solar activity is simulated, is described by Blake \& Lewis (2014). Instead of repeating an in-depth analysis of the origins of this inherent variability, this paper will focus on analysing its impact on the results of DAMAGE, and thus similar models. It will also quantify the uncertainty of the predictions of the number of objects in orbit and the number of collisions in one specific scenario, which was also used in the IADC comparison study.

\section{Future number of objects in orbit}

In order to understand how many Monte Carlo runs are needed to generate a large enough sample, which enables the variability of the used debris model to be captured reliably, a Monte Carlo projection using 25000 runs was carried out. This number of MC runs was at least 90 times greater than used in the IADC comparison study (Liou et al., 2013) (at most 275 runs were used in the quoted study). DAMAGE was used to study the possible outcomes of implementing strict debris mitigation measures, which is one of the commonly considered options to reduce the growth rate of the number of objects in orbit. The settings for this "mitigation only" scenario are summarised in Table 1. They are based on the settings used to generate the DAMAGE projection for the IADC comparison study (Liou et al., 2013). One notable difference between the DAMAGE settings and other projections in the study by Liou et al. (2013) is the simulation of the post-mission disposal compliance rate. Here, the $90 \%$ compliance was applied to all spacecraft in LEO, whereas other models in the IADC comparison study applied it to only the payloads with nominal lifetimes greater than 25 years. Despite this difference, the increase in the number of objects by 2209 predicted with DAMAGE agreed to within $10 \%$ with respect to the mean of all the models (Liou et al., 2013). The only modification that was made to the "mitigation only" scenario used here, which was not included in the work by Liou et al. (2013), was simulation of collision avoidance by active spacecraft. This is to say that for the first 8 years in orbit, i.e. during their operational lifetimes, spacecraft could not be involved in collisions. Inclusion of this debris mitigation measure reduces the mean number of objects in 2209 by $6.1 \%$ and the number of catastrophic collisions observed over 200 years by $13.3 \%$ when compared to implementing all other debris mitigation measures, which were included in the work by Liou et al. (2013) (Lewis et al., 2012).

The evolution of the number of objects larger than $10 \mathrm{~cm}$ obtained by 


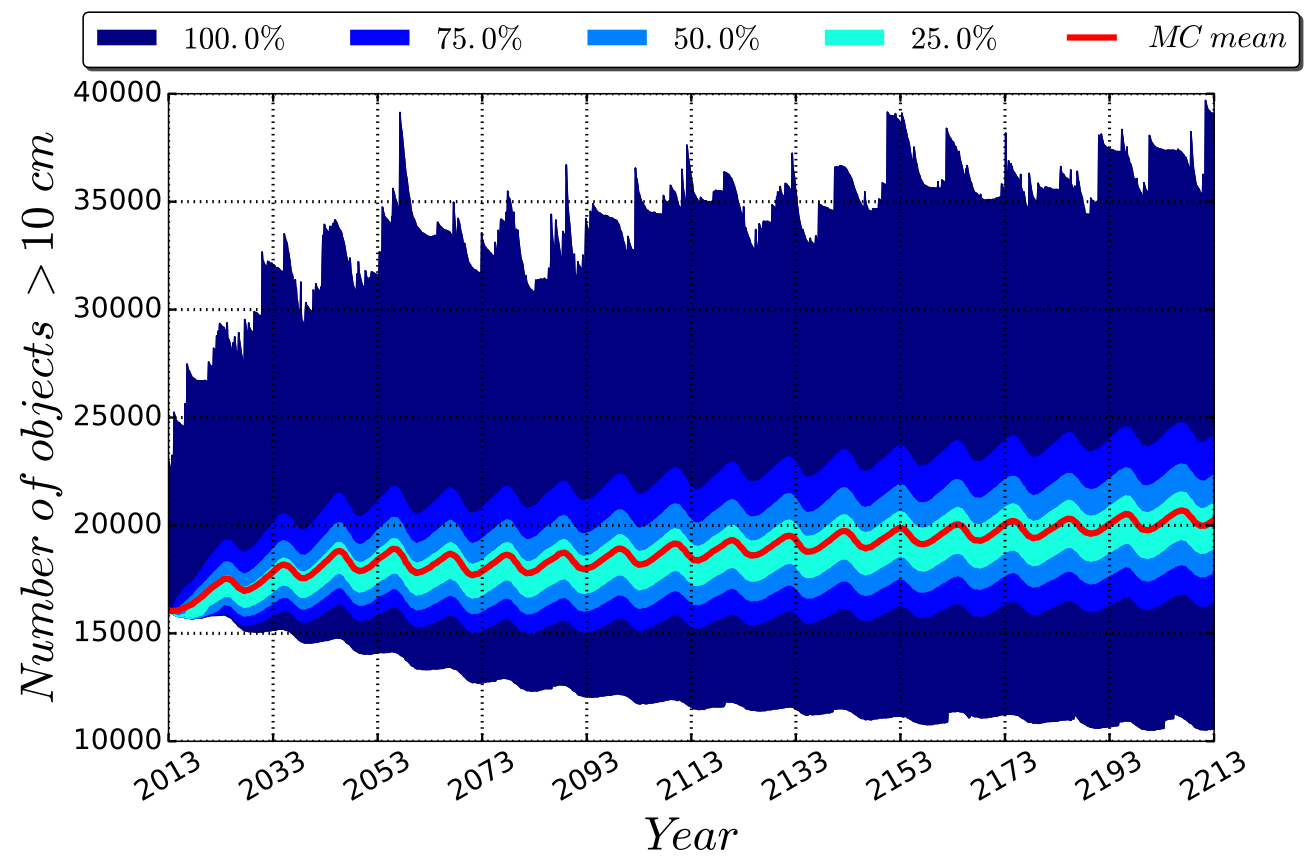

Figure 1: Evolution of the number of objects larger than $10 \mathrm{~cm}$ in the Monte Carlo sample for the "mitigation only" scenario, summarised in Table 1. Showing the number of objects in a given fraction of the Monte Carlo runs, centred on the median. Mean of the sample is also shown. The scenario was simulated using $25000 \mathrm{MC}$ runs.

projecting the "mitigation only" scenario from Table 1 is shown in Fig. 1; in order to represent the evolution of the probability density in the entire MC sample over time, different fractions of the runs, centred around the median, were plotted. Several statistical parameters, describing the shape of the distribution of the number of objects at the end of the projection (in the end of 2213), are summarised in Table 2.

The mean number of objects increases. The same behaviour was observed by Liou et al. (2013). However, considerable spread away from the mean can be observed in Fig. 1, which was also observed by Liou \& Johnson (2008). In $9.5 \%$ of the cases, the number of objects did not increase by the end of the projection period c.f. the initial population size. This means that, under the launch traffic, solar activity and post-mission disposal (PMD) compliance 
Table 1: Simulation settings for DAMAGE used in an IADC comparison study, which followed the previous study by Liou et al. (2013), and in this work. The scenario is titled by the name it is referred to in text and in figures. It is supposed to investigate the outcome of trying to preserve the debris environment by not polluting it any further, i.e. good compliance with debris mitigation.

\begin{tabular}{r||c}
\multicolumn{1}{c||}{ Parameter } & "Mitigation only" \\
\hline \hline $\begin{array}{r}\text { Fraction of all LEO } \\
\text { objects that comply with } \\
\text { post-mission disposal } \\
\text { guidelines }\end{array}$ & $90 \%$ \\
\hline Simulation start epoch & 1 Jan 2013 \\
\hline Simulation end epoch & Dec 2213 \\
\hline Modelled launch traffic & Repeated 2005-2012 launch traffic \\
\hline Modelled solar activity & $\begin{array}{r}\text { Repeated average pseudo-sinusoidal } \\
\text { cycle, details given in (Lewis \& } \\
\text { Horbury, 2013) }\end{array}$ \\
\hline $\begin{array}{r}\text { Modelled objects } \\
\text { distance }\end{array}$ & $\begin{array}{r}\text { Objects larger than } 10 \text { cm from } \\
\text { MASTER 2013 reference population }\end{array}$ \\
\hline $\begin{array}{r}\text { Time step at which close } \\
\text { approaches are found to } \\
\text { simulate collisions }\end{array}$ & $\sqrt{300} \approx 17.32 \mathrm{~km}$ \\
\hline $\begin{array}{r}\text { Spacecraft operational } \\
\text { lifetime }\end{array}$ & 5 days \\
\hline
\end{tabular}




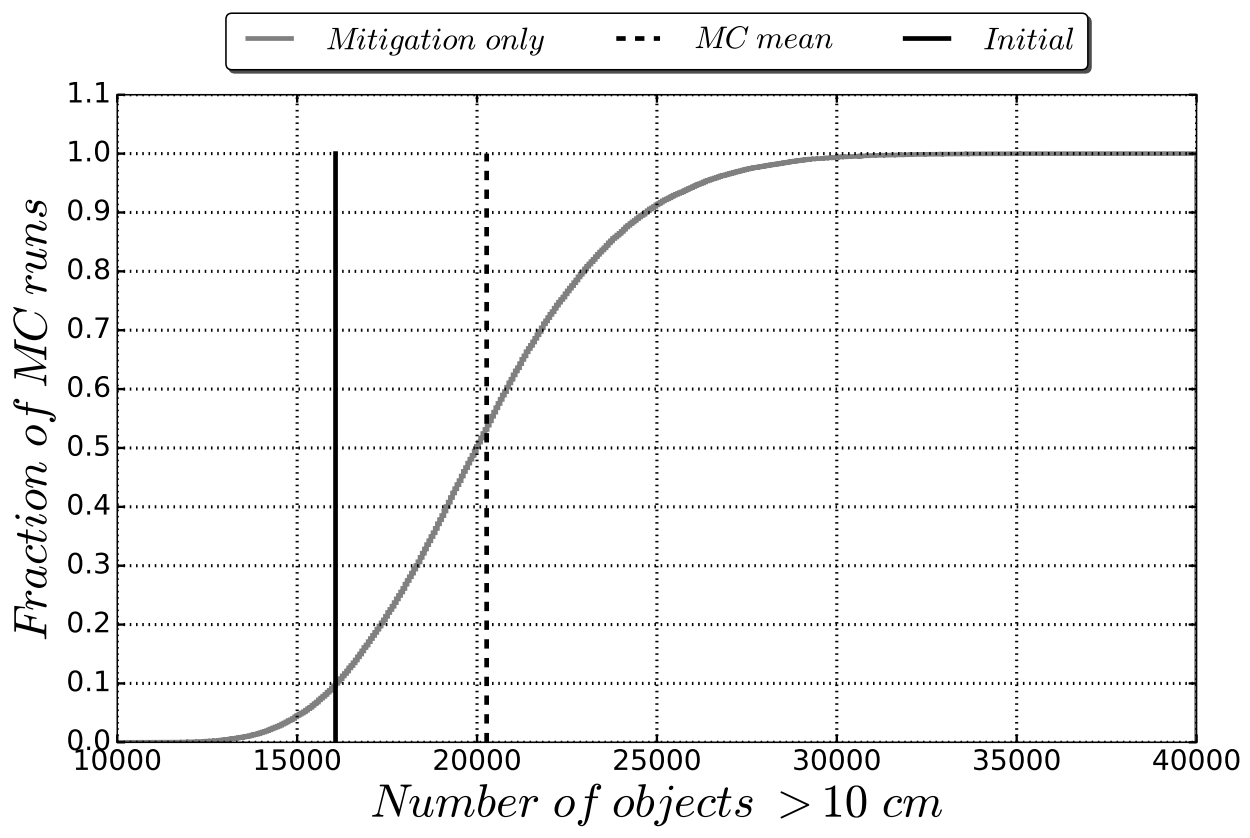

Figure 2: Cumulative histogram showing the fraction of the MC runs where the number of objects in 2213 (end of the projection) was less than a given value. Also showing the mean of the MC sample at that epoch, and the initial number of objects in 2013. "Mitigation only" scenario from Table 1.

assumptions, mitigation only would be sufficient to prevent the increase in the number of objects in orbit. Notably, the mean of the sample is always above the median, which is in the middle of the lightest-blue region in Fig. 1. Figure 2 shows that for $53.0 \%$ of the Monte Carlo runs, the number of objects at the end of the projection was less than the mean, which was 20267 objects.

The large variability in the MC sample raises concerns whether sufficiently many Monte Carlo runs were used to reliably capture the possible future distribution of the objects. If the number of Monte Carlo runs was sufficient, the distribution of the number of objects should not change if more MC runs are added into the sample.

Liou (2008) and White \& Lewis (2014) compared the mean numbers of objects obtained with various Monte Carlo sample sizes to the means obtained with samples consisting of 200 projections. In their analyses, there 
Table 2: Statistical parameters of the distribution of objects larger than $10 \mathrm{~cm}$ in the Monte Carlo sample of 25000 runs in 2213. "Mitigation only" scenario from Table 1.

\begin{tabular}{c||c} 
Distribution parameter & Value \\
\hline \hline Mean (no. objects) & 20267 \\
Median (no. objects) & 20019 \\
Variance (no. objects $^{2}$ ) & 11402561 \\
Skewness (unitless) & 0.4727 \\
Kurtosis (unitless) & 0.3080
\end{tabular}

was a probability of over $85 \%$ that 10 to $40 \mathrm{MC}$ runs were sufficient to yield a mean number of objects within $10 \%$ of the mean of the $200 \mathrm{MC}$ runs (Liou, 2008). However, no analysis has been performed to ascertain whether this reference mean was reliable, i.e. whether the empirical distribution used in the Monte Carlo bootstrap method was accurate (DeGroot \& Schervish, 2014).

Similarly, no data have been presented that would show how other parameters of the distribution of the future projections vary with the number of Monte Carlo runs in the sample. This is important because "mitigation only" may be sufficient to prevent the growth of the objects in orbit. Ensuring that the distribution of the future projections is well captured by the MC sample will enable the possible outcomes of "mitigation only" to be assessed meaningfully. Providing the uncertainty information to the decision makers will make it possible for them to trade off considerable costs (financial, political etc.) of performing space debris remediation against implementing imperative and stricter mitigation measures, for example.

\section{Sufficient number of Monte Carlo runs}

The data about the number of objects larger than $10 \mathrm{~cm}$ in 2213 from the "mitigation only" scenario in Table 1 were analysed. The relationship between the shape of the final distribution of the number of objects in the Monte Carlo sample and the number of runs in this sample was investigated. The analysed shape parameters were the arithmetic mean, variance, skewness, kurtosis, and median. These are the first four moments of the distribution that can be used to describe its shape, as well as the value that divides the probability space in two halves (DeGroot \& Schervish, 2014). Here, the value 
of the kurtosis of the normal distribution was zero, i.e. Fisher kurtosis was computed (Scipy-Stats, 2015).

The bootstrap technique identical to that by Liou (2008) was used, however with all the mentioned statistical parameters of the distribution, not only the MC mean. Varying numbers of Monte Carlo runs were used as the complete sample, starting at 10 and finishing at 25000 with increments of 50. For each MC sample size, the individual MC runs were randomly selected from the pool of 25000 and the mentioned statistical parameters of the distribution recomputed. This random selection was repeated 2000 times, thus giving distributions of the shape parameters for every sample size. Percentiles of these distributions were computed, the MC sample size was incremented and the process repeated until all desired sample sizes were investigated.

The hypothesis of this investigation is that when a sufficient number of MC runs is present in the sample, the individual runs should not significantly affect the distribution of the number of objects. Consequently, each of the shape parameters should reach a steady, distinct value when the number of MC runs is large enough. The evolution of the mean number of objects with the number of Monte Carlo runs in the sample is shown in Fig. 3. The variations of the median and variance are given in Fig. 4, whereas Fig. 5 shows how the skewness and kurtosis of the distribution depend on the size of the sample.

More Monte Carlo runs were needed to estimate the higher-order moments of the distribution than the mean. Sample size of $60 \mathrm{MC}$ runs was needed for the means of all of the 2000 random selections of the MC runs to be within $10 \%$ of the mean estimated using 25000 runs. $60 \mathrm{MC}$ runs were needed to estimate the median with the same precision. Sample sizes of 3560, 16060 and 23960 runs were needed to ensure that variance, skewness and kurtosis were within $10 \%$ of the value estimated using 25000 runs.

Skewness shown in Fig. 5a can be both positive and negative for Monte Carlo samples smaller than approximately 400 runs. This means that, with such sample sizes, it is impossible to discern whether the distribution of the number of objects has a tail (outlying runs) above or below the mean (DeGroot \& Schervish, 2014). At least 410 MC runs were needed to ensure that the skewness wasf non-negative.

The same bootstrap technique was performed but population snapshots were examined at different census epochs. The results of this study are presented in Table 3. Means and medians of the Monte Carlo samples could 
always be estimated with relatively small samples of 60 runs, which confirms the findings of other authors (Liou \& Johnson, 2006). There is a clear trend in more $\mathrm{MC}$ runs being required to estimate the higher orders of the distribution at all the investigated census epochs, which corroborates the findings of the analysis of the distribution in 2213. More MC runs were needed to estimate the sample skewness and kurtosis at census epochs later than 2063. However, more runs were needed to estimate the sample variance in 2063 than at later census epochs. These relationships between sample sizes required to reach a given level of precision and census epochs may not be definitive because only four census epochs were analysed. A sensitivity study to the location of the census epochs and the number of repetitions in the bootstrap would need to be performed in order to enable drawing binding conclusions on the relationship between the minimum sample size and the length of the projection. This was not performed due to computational time restrictions, as discussed in Section 7. However, the relationship between the sample size, census epoch, and precision of estimating different moments of the distribution will be qualitatively discussed in Section 7

Several conclusions may be drawn from the presented investigation. Firstly, the findings of Liou \& Johnson (2006) were corroborated and similar numbers of $\mathrm{MC}$ runs were needed to estimate the means of the MC samples with $10 \%$ precision. However, more Monte Carlo runs were needed to estimate higher moments of the distribution, i.e. variance, skewness and kurtosis, with the same precision. Also, the higher the precision on the given distribution parameter is required, the more MC runs are needed. The Monte Carlo sample should be large enough so that the probability density in its tail is low, in order to avoid situations where one additional run with a large final number of objects will change the parameters of the distribution. Lastly, the skewness of the distribution was found to be positive. This indicates that the distribution of the number of objects is not normal, which is investigated in more detail in Section 5.

\section{Analysis of the final distributions}

The positive skewness shown in Fig. 5a indicates that this distribution of the number of objects has a tail above the mean. In other words, the MC runs

with the highest final number of objects are further away from the mean of the distribution than the runs with the fewest objects. This is also visible in Fig. 1, which shows that certain MC runs had over twice as many objects as 


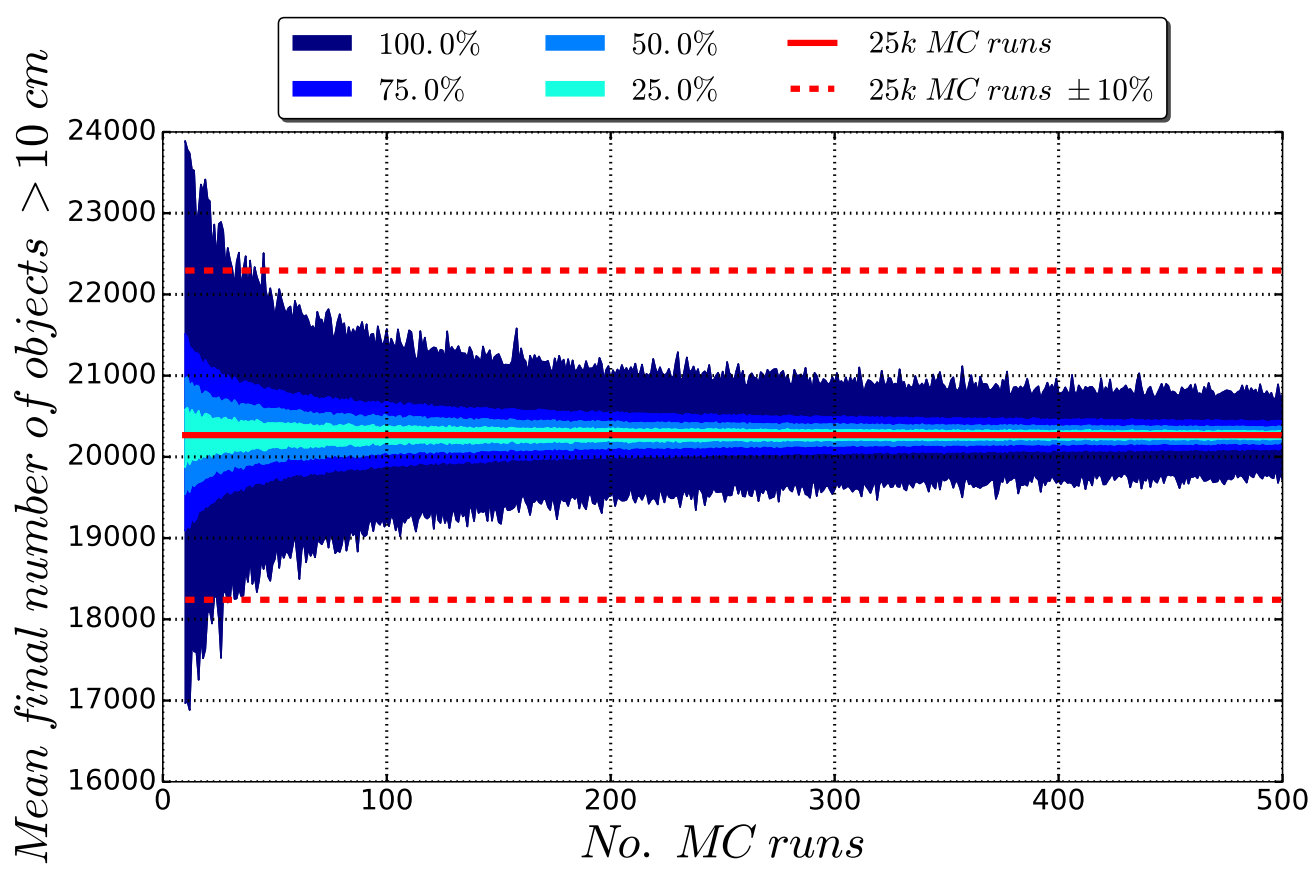

Figure 3: Variation of the arithmetic mean of the number of objects in the MC sample of the "mitigation only" scenario from Table 1 at the end of the projection (2213) with the sample size. Monte Carlo runs corresponding to the given sample size were randomly selected 2000 times, and the indicated percentiles of the distribution computed for every sample size. Also showing the value obtained with $25000 \mathrm{MC}$ runs, $\pm 10 \%$. 


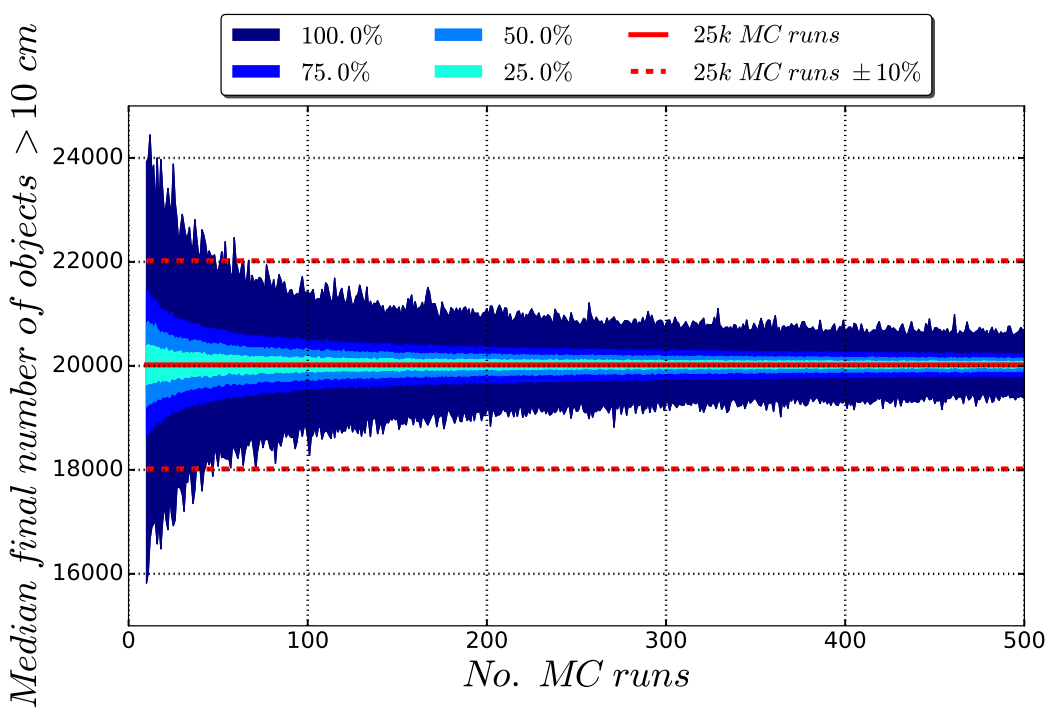

(a) Median

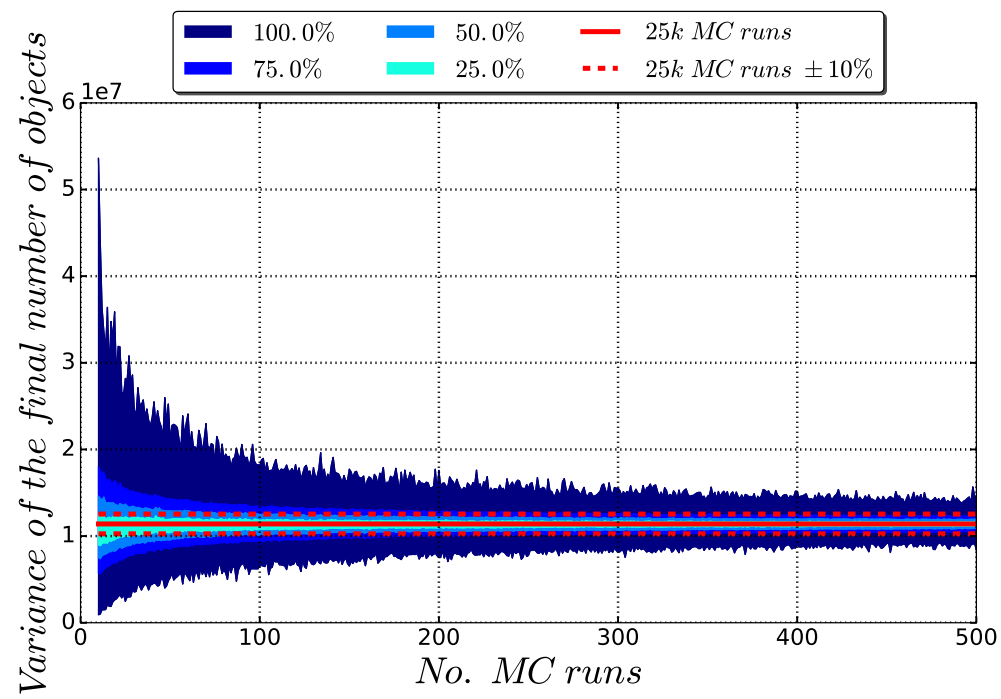

(b) Varaince

Figure 4: Variation of the median and variance of the number of objects in the MC sample of the "mitigation only" scenario from Table 1 at the end of the projection (2213) with the sample size. Monte Carlo runs corresponding to the given sample size were randomly selected 2000 times, and the indicated percentiles of the distribution computed for every sample size. Also showing the value obtained with $25000 \mathrm{MC}$ runs, $\pm 10 \%$. 


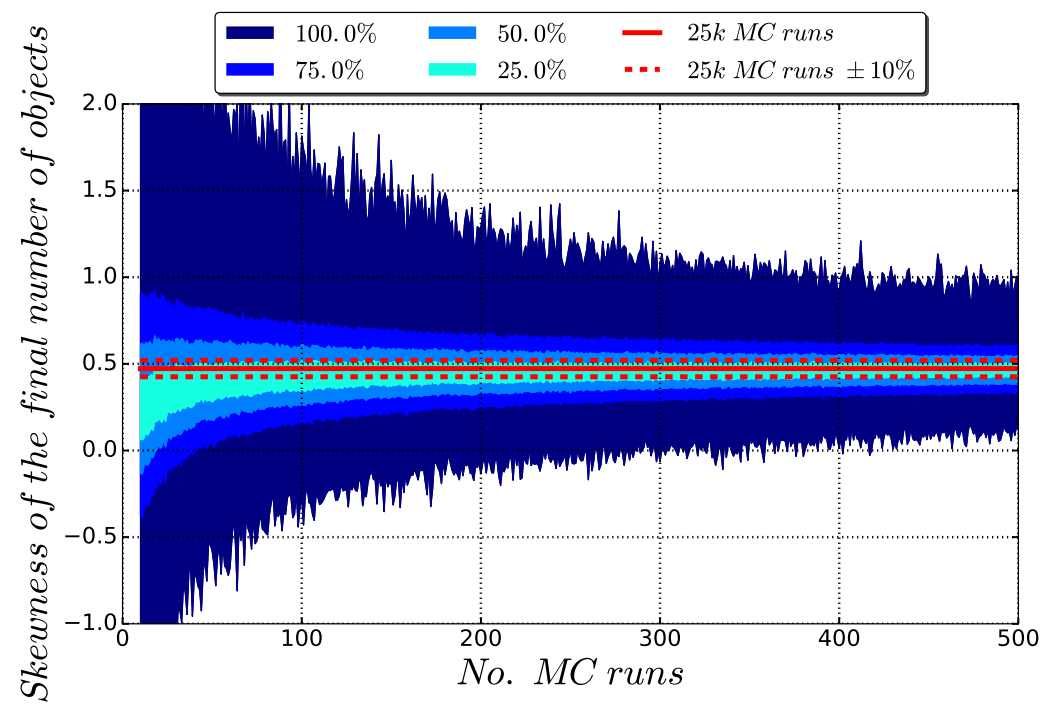

(a) Skewness

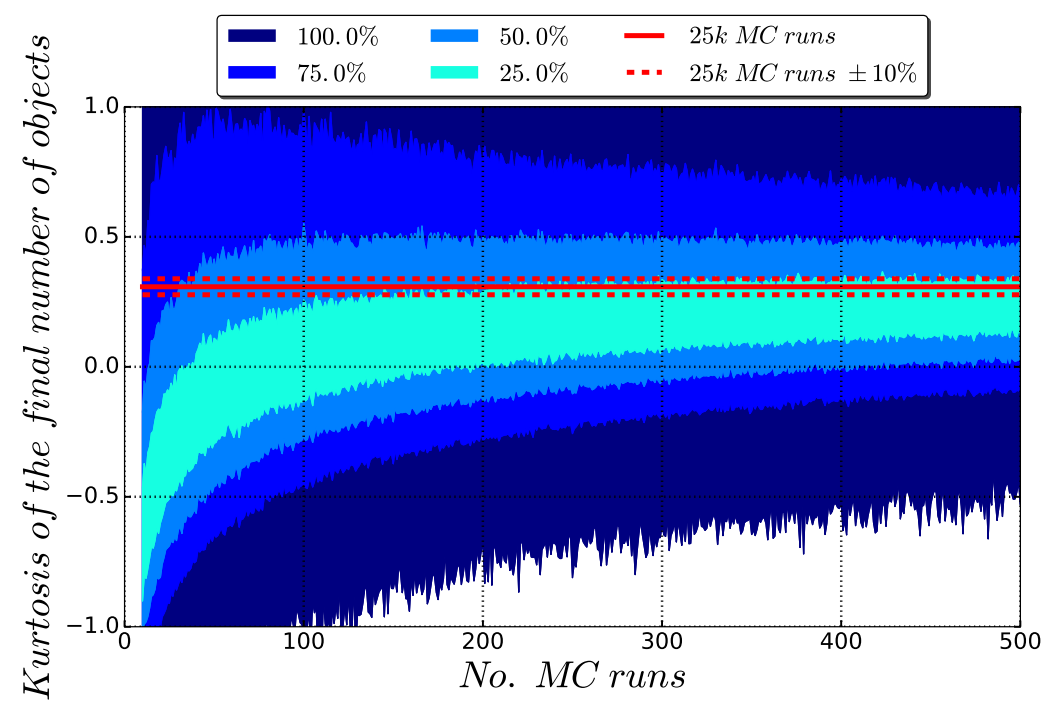

(b) Kurtosis

Figure 5: Variation of the skewness and kurtosis of the number of objects in the MC sample of the "mitigation only" scenario from Table 1 at the end of the projection (2213) with the sample size. Monte Carlo runs corresponding to the given sample size were randomly selected 2000 times, and the indicated percentiles of the distribution computed for every sample size. Also showing the value obtained with $25000 \mathrm{MC}$ runs, $\pm 10 \%$. 
Table 3: MC sample size required to estimate a given parameter of the distribution of the number of objects with precision of $10 \%$ w.r.t. 25000 runs at different census epochs. "Mitigation only" scenario from Table 1. The MC sample sizes are estimated to within 50 runs.

\begin{tabular}{c||rrrr}
\multicolumn{1}{c||}{ Distribution parameter } & \multicolumn{4}{c}{ Census epoch } \\
& $\mathbf{2 0 6 3}$ & $\mathbf{2 1 1 3}$ & $\mathbf{2 1 6 3}$ & $\mathbf{2 2 1 3}$ \\
\hline \hline Mean & 60 & 60 & 60 & 60 \\
Median & 60 & 60 & 60 & 60 \\
Variance & 4260 & 3260 & 3460 & 3560 \\
Skewness & 13510 & 14260 & 14860 & 16060 \\
Kurtosis & 22160 & 23160 & 24010 & 23960
\end{tabular}

the mean, whereas none of the runs had half the number of objects. Finally, more MC runs had the number of objects below than above the mean, as can be seen in Fig. 2.

This raises a question about how the uncertainty in the results should be represented. If the distribution is not normal then using mean and standard deviation to represent the results, as is commonly done (Liou \& Johnson, 2006; DoladoPerez et al., 2015; Radtke \& Stoll, 2016), may not convey all the uncertainty information. The final distribution of the number of objects was therefore analysed further.

The normal probability plot of the final distribution of the number of objects in the Monte Carlo sample is shown in Fig. 6. The distribution is not normal, i.e. it is not a straight line on the normal probability plot in Fig. $6(R=0.9937)$. The presence of a tail above the mean suggests that the distribution might be log-normal, thus the probability plot for this type of distribution was created and is shown in Fig. 7. The distribution has a higher correlation $(R=0.9999)$ on this probability plot, thus it is more likely that the distribution is log-normal than normal (DeGroot \& Schervish, 2014).

A physical explanation for this phenomenon is that several large collision events close to the final simulation epoch or at high altitudes are sufficient for the number of objects in a given Monte Carlo run to be high. On the other hand, it is difficult for the number of objects to be low. This requires collisions to occur only at low altitudes, where the atmospheric drag is high enough to cause the resulting debris to decay by the end of the simulation, or for all the collisions to generate few fragments. The presence of outliers with 


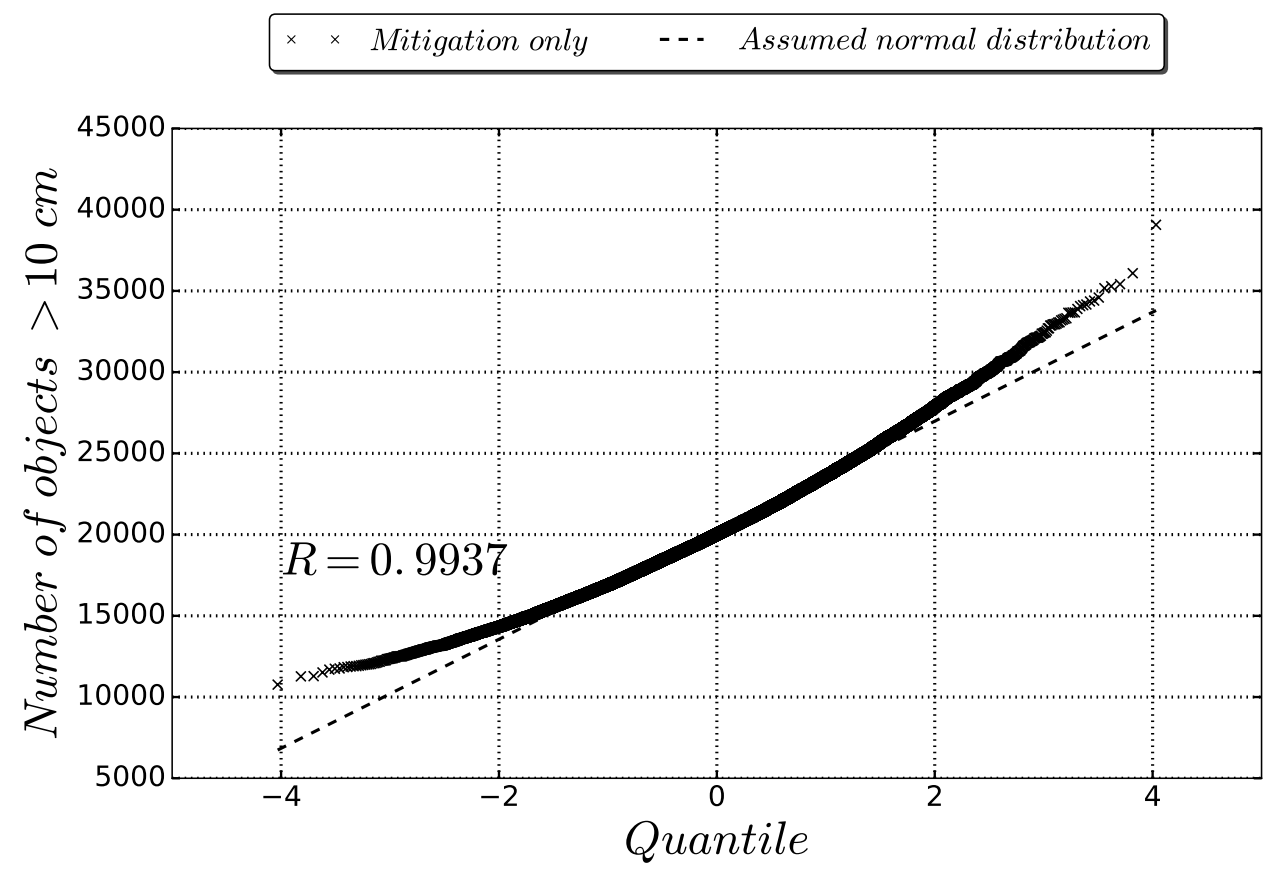

Figure 6: Probability plot of the number of objects larger than $10 \mathrm{~cm}$ in the MC sample at the end of the projection (2213). Compared to theoretical normal distribution and showing how well the data match the assumed distribution. "Mitigation only" scenario from Table 1 projected with 25000 Monte Carlo runs.

a high number of objects will increase the mean of the sample and place it above the median. This means that the probability density below the mean is higher than at the mean. Thus, there is a higher probability that the number of objects will be lower than the mean, rather than higher $(53.0 \%$ of the Monte Carlo runs had fewer objects than the mean in this study).

\section{An alternative figure of merit}

The previous discussion in this paper analysed the distributions of the numbers of objects $\geq 10 \mathrm{~cm}$ passing through the LEO region. This is consistent with the way the results of evolutionary debris models were presented e.g. in the IDAC comparison study (Liou et al., 2013). It was investigated 


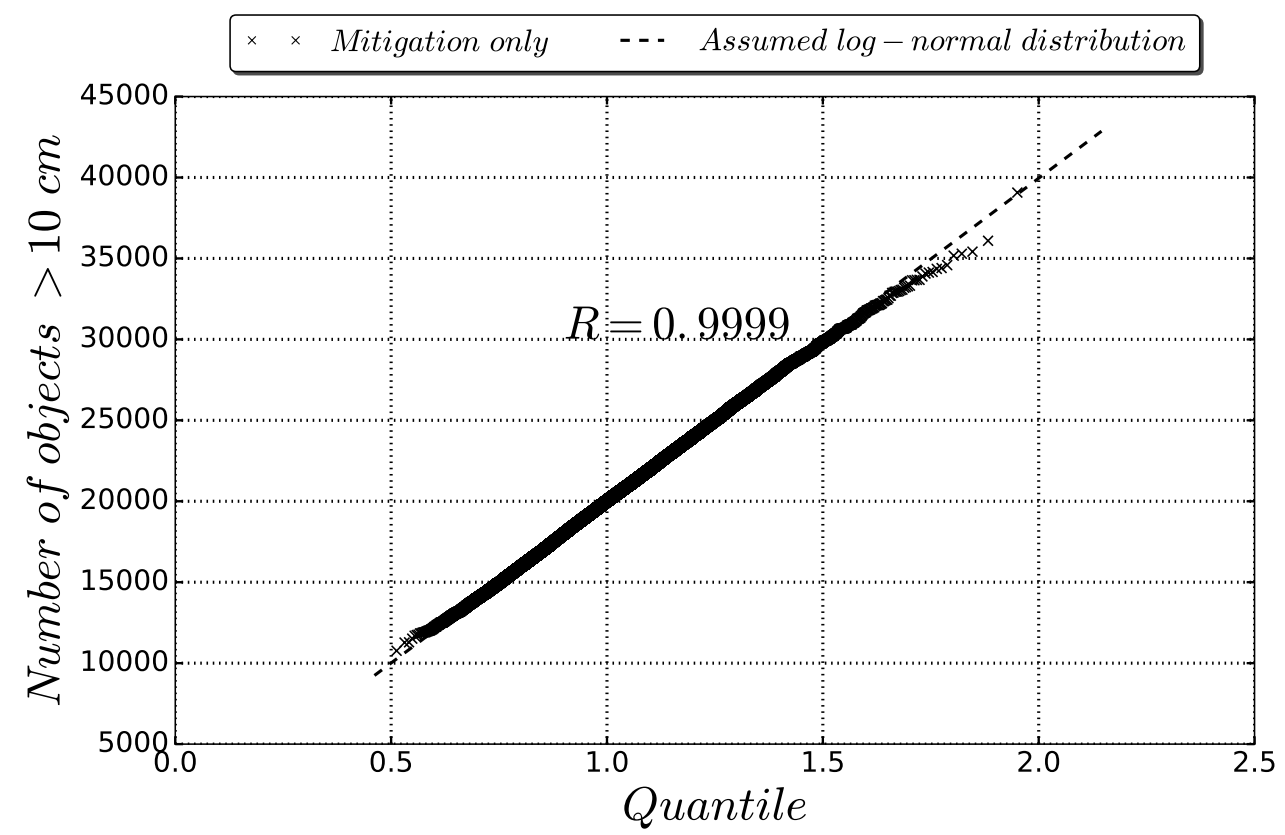

Figure 7: Probability plot of the number of objects larger than $10 \mathrm{~cm}$ in the MC sample at the end of the projection (2213). Compared to theoretical log-normal distribution and showing how well the data match the assumed distribution. "Mitigation only" scenario from Table 1 projected with 25000 Monte Carlo runs. 
whether using a different figure of merit describing the evolution of the debris environment would require as many $\mathrm{MC}$ runs to reliably capture the distribution across the Monte Carlo sample, which could offer computational time savings for future studies. The number of catastrophic collisions that took place over 200 years in every MC run was found. This distribution of the number of collisions that occurred until 2213 is shown in Fig. 8. The distribution does not have considerable tails above or below the mean. This means that the underlying distribution could not be log-normal, unlike the distribution of the number of objects in 2213. To test the type of the underlying distribution, probability plots were created to compare the empirical distribution of the number of catastrophic collisions in the $\mathrm{MC}$ sample to normal and log-normal theoretical distributions, and are shown in Fig. 9. Both theoretical distributions appear equally likely with $R=0.9961$ for normal and $R=0.9960$ for log-normal. The fact that the mean (33.56) obtained with $25000 \mathrm{MC}$ runs was higher than the median (33.0) suggests that the distribution could be log-normal. However, this is not as profound as in the case of the number of objects.

To ensure that the MC sample was large enough to correctly represent the number of collisions, a bootstrap study identical to that from section 4 was undertaken and the results are shown in Fig. 10. As before, more runs were needed to estimate the higher orders of the distribution. 60 and $110 \mathrm{MC}$ runs were sufficient for the mean and the median to converge within $10 \%$ of the value obtained with $25000 \mathrm{MC}$ runs. For variance, skewness and kurtosis the sample sizes that gave the same precision were 3860, 19360 and 24360 , respectively.

Median number of collisions was varying between 25.5 and 43.0, and all the permutations of the MC runs reached the median of 33.0 with sample size of 6510 runs. $75 \%$ of all the bootstrap permutations reached this value with sample sizes of 1060 runs. The remaining $25 \%$ of permutations were outliers with medians of 32.0 and 34.0. The presence of these outliers manifests itself as "spikes" and "plateaus" in Fig. 10. Such variation can also be seen in all other bootstrap plots presented here, it is only the scale of the median plot that amplified this variability for this parameter. Such variability is to be expected, and it is the reduction in this variability that the bootstrap technique aims to demonstrate as the MC sample increases. The fact that this variability reduced in the bootstrap on the median number of collisions as the sample size increased, signifies that this bootstrap was performed correctly. 


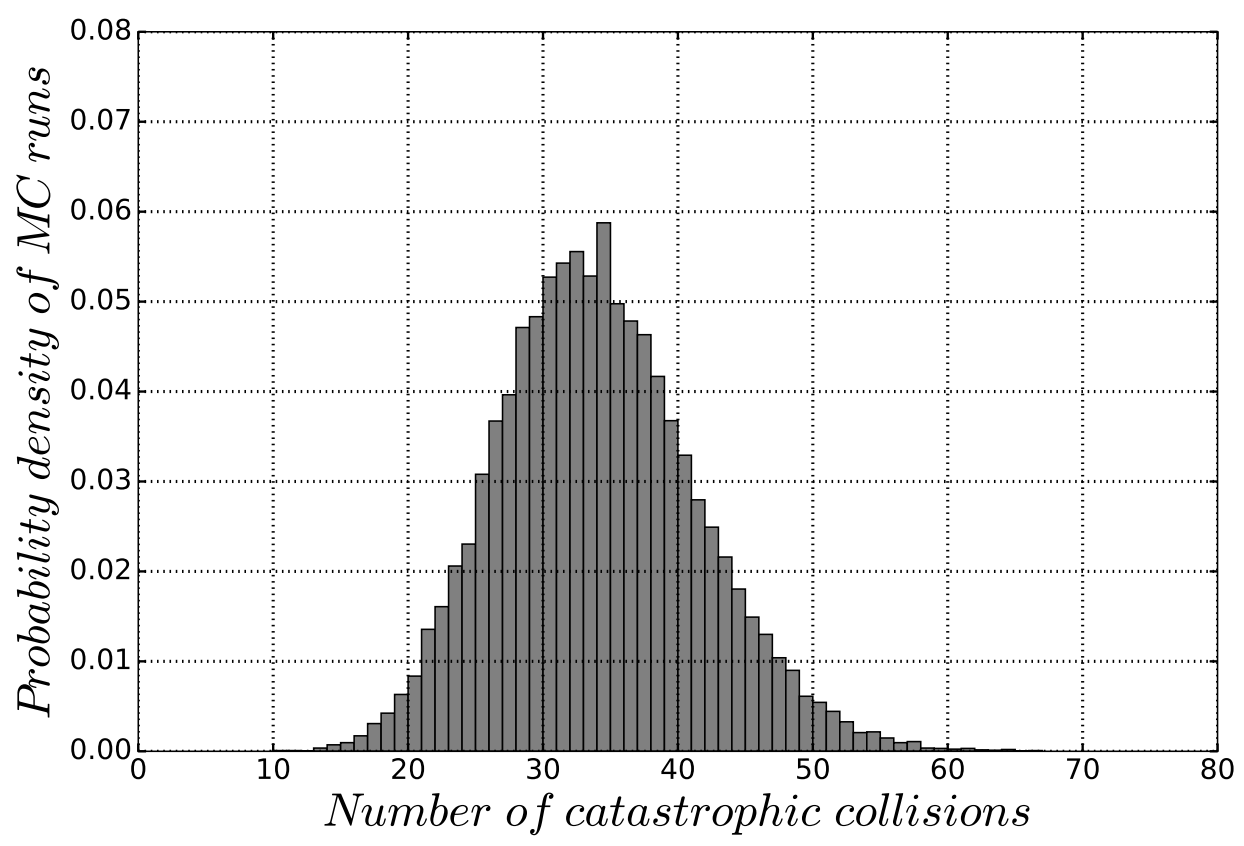

Figure 8: Histogram of the number of catastrophic collisions that occurred until 2213. "Mitigation only" scenario from Table 1 projected with 25000 Monte Carlo runs.

Overall, using the number of catastrophic collisions to, for example, compare different debris mitigation measures does not appear to offer any computational time savings over examining the number of objects $\geq 10 \mathrm{~cm}$. This is because similar sizes of the Monte Carlo samples (same order of magnitude) were needed to achieve the same level of precision with respect to the distribution parameters obtained with 25000 Monte Carlo runs in both cases.

\section{Discussion}

The uncertainty in the number of objects in orbit may not have been well captured by previous space debris environment studies due to their relatively small MC sample sizes. This does not need to be the case if the number of runs in those studies happened to give a precise representation of the final distribution of the number of objects. However, this cannot be guaranteed 


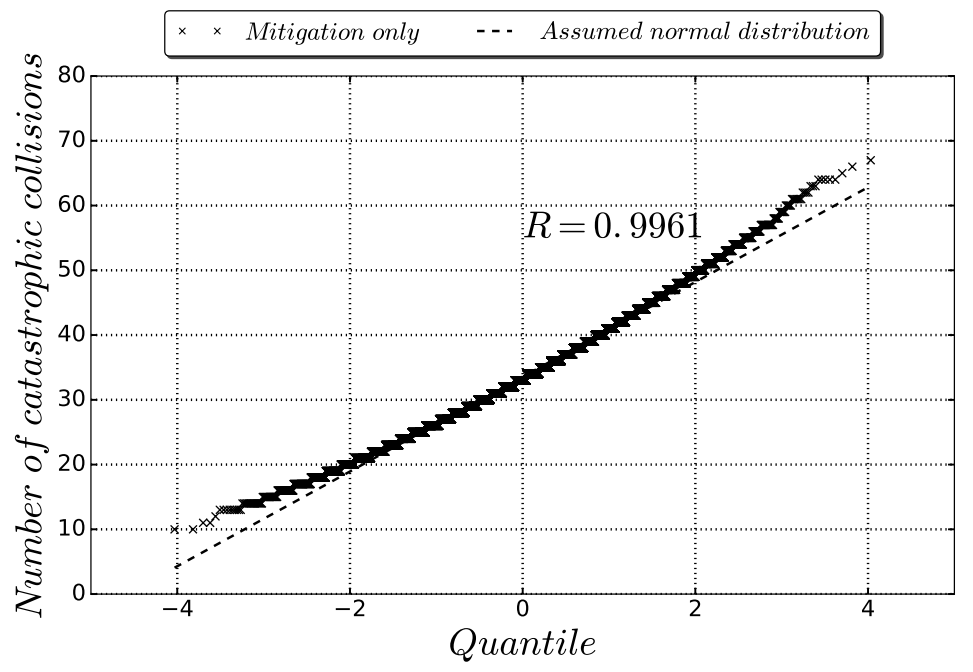

(a) Normal

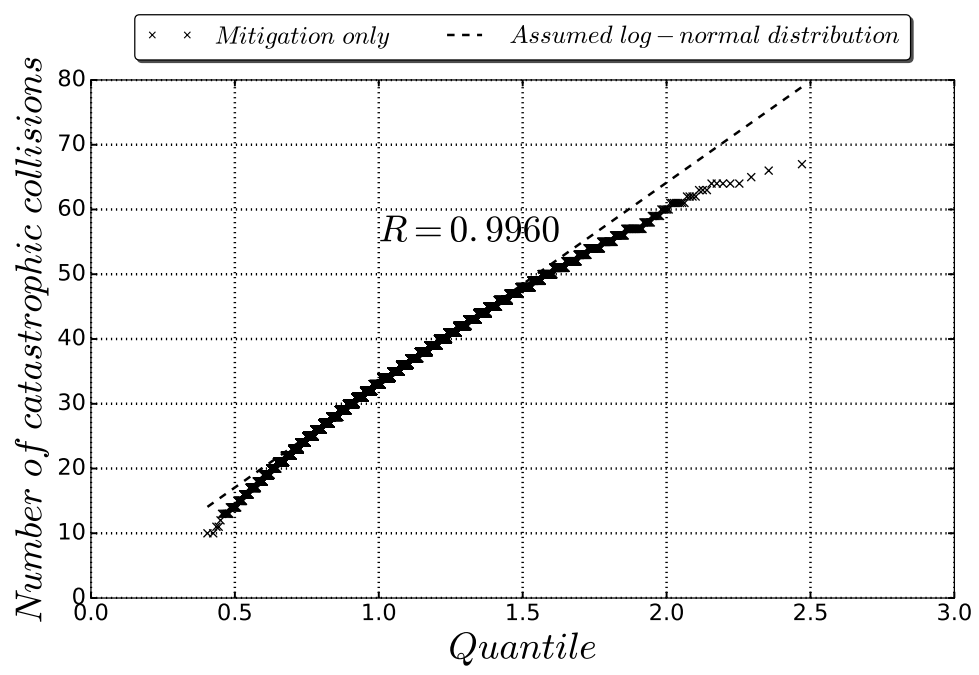

(b) Log-normal

Figure 9: Probability plots of the number of catastrophic collisions that occurred until 2213. Comparing the empirical distribution to assumed theoretical log-normal and normal distributions. "Mitigation only" scenario from Table 1 projected with 25000 Monte Carlo runs. 


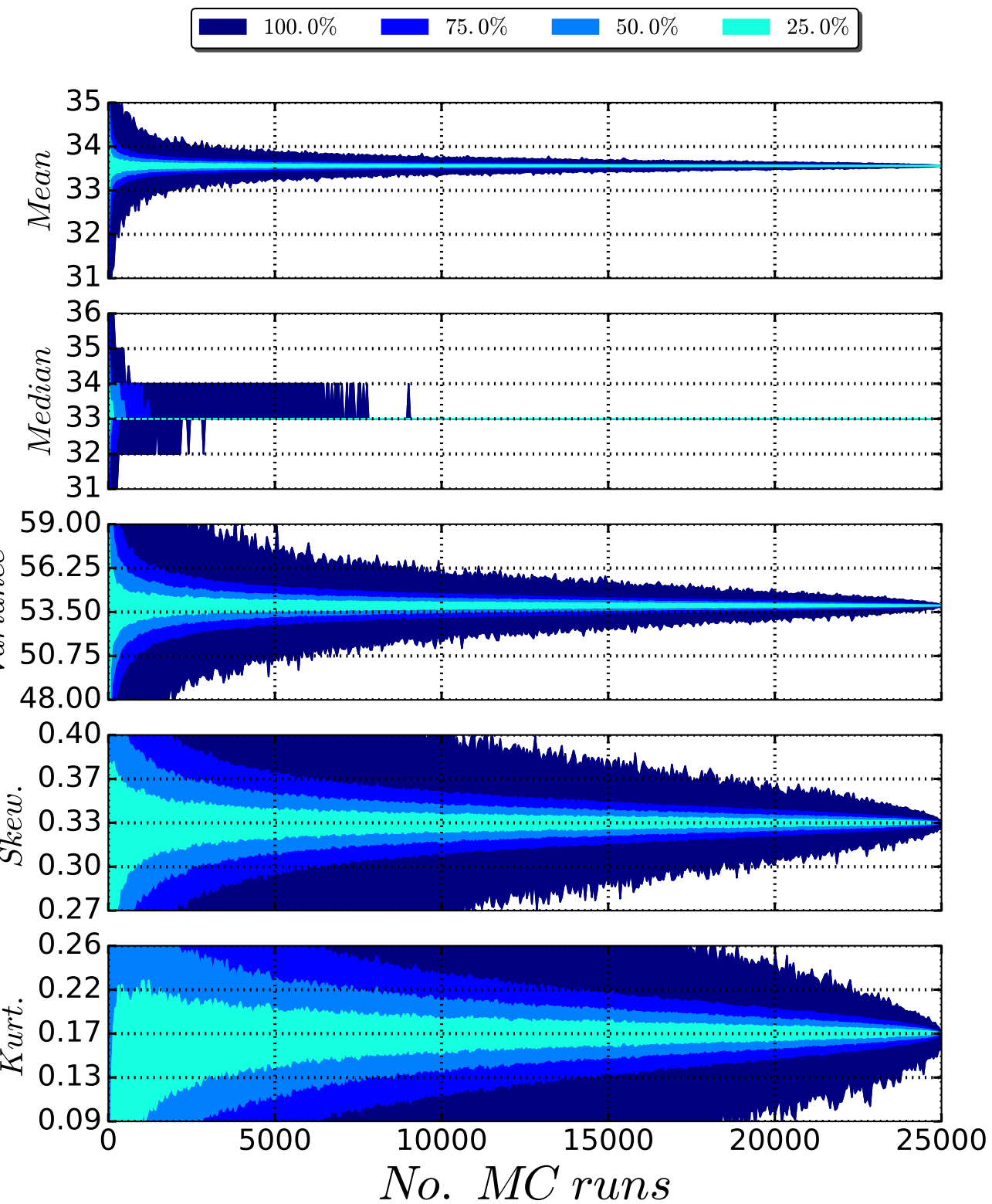

Figure 10: Variation of the mean, median, variance, skewness and kurtosis of the number of catastrophic collisions that occurred until 2213 with the sample size. "Mitigation only" scenario from Table 1, details given in text. 
with such small samples. Moreover, smaller Monte Carlo samples may not accurately represent the probability density of the number of objects, even if the parameters of the distribution are accurately estimated.

It cannot be guaranteed that the distribution of the number of objects obtained here would not vary if more the 25000 Monte Carlo runs were used. The uncertainty on the value of the obtained moments of the distribution is the larger the higher the order of the moment of the distribution. However, as shown in Fig. 11, the variation in the mean, median, variance and skewness reduced considerably when more than 500 runs were already present in the sample, c.f. Fig. 3, Fig. 4, and Fig. 5. However, 24983 out of $25000 \mathrm{MC}$ runs were needed to estimate kurtosis of the number of objects with precision of $10 \%$. The results of this bootstrap on kurtosis with sample sizes from 24900 to $25000 \mathrm{MC}$ runs are shown in Fig. 12. The distributions of kurtosis are well within the upper precision bound even for smaller samples, but they have a tail below the mean value, which extends to approximately the lower precision bound. This demonstrates that more runs might be needed to ensure the distribution of kurtoses is narrower, i.e. does not have a prominent tail. Similarly, a bootstrap on the number of random MC runs selected for every sample size would be necessary to ensure that the distributions of the shape parameters are captured reliably. However, it is the distribution of the number of objects at the census epoch that is of prime interest. As shown in Fig. 13 and Fig. 14, the probability density in the tails of the distributions obtained with $25000 \mathrm{MC}$ runs were relatively low at all census epochs. Thus, it is extremely unlikely that larger outliers, which could change the shapes of the distributions, would be encountered if a larger sample was used. This is because the probability of generating such runs is very low, albeit nonzero. Therefore, the distribution of the number of objects is believed to be well captured, but the recommended sample sizes to reach a given level of precision should be used as a guideline only.

The uncertainty about the parameters of the distributions of the number of objects obtained here also extends to classification of these distributions as log-normal rather than normal. However, in this case, a physical phenomenon was identified that would give rise to such a behaviour of the number of objects. Also, several premises, hinting at the type of the distribution, were identified and thus it is believed that the distribution is log-normal. However, the nature of the distribution changes with the length of the projection. Every Monte Carlo run has the same initial number of objects, i.e. the initial distribution is uniform. Collisions need to occur in the MC runs for 
the tail above the mean to appear and the distribution to become log-normal. Collisions are simulated based on random numbers and thus the earlier the census epoch, the fewer collisions have taken place in the $\mathrm{MC}$ sample and the less log-normal the distribution could be.

However, collisions do occur early in the projection in certain MC runs. By 2063, enough collisions took place to increase the number of objects in those runs considerably above the mean, as can be seen in Fig.14b. The maximum number of objects in 2063 was 33 367, i.e. only $17 \%$ different than the maximum number of objects recorded in 2213 (39 068). These outlying runs increased the variance of the sample shortly after the start of the projection. They also made the distribution asymmetric, with few outlying runs being present in the tail, which was associated with high skewness and kurtosis at the beginning of the projection. When collisions took place in the rest of the MC runs in the sample, more MC runs had a large number of objects, the distribution became less asymmetric and so the the skewness and kurtosis decreased, as shown in Table 4. This evolution of the tail of the distribution can also be observed by comparing all the subplots in Fig. 13 and Fig. 14. Because the probability density in the tail was low in 2063, many MC runs were needed to estimate the variance of the sample at that epoch, which was reported Section 4 (the probability of generating runs with a high number of objects was low). For later census epochs, the distribution was less asymmetric with a less distinct peak, and so fewer runs were needed to estimate the sample variance (probability of generating MC runs with a large number of objects was higher). More runs seem necessary to estimate skewness and kurtosis of the distribution at later census epochs, which was also reported in Section 4. However, the variability between the sample sizes needed to reach a given level of precision for these moments of the distribution was lower than in case of variance, and so this trend could have been an artefact stemming from the location of the census epochs. Variance varied by $31 \%$ between the different census epochs analysed in Section 4, whereas skewness and kurtosis varied by $19 \%$ and $8 \%$, respectively. The location of the census epochs definitely affected the behaviour observed for variance. Still, a MC run with a relatively large number of objects, which would increase the sample variance, could appear arbitrarily close to the beginning of the projection. Because the probability of generating such runs is low, large MC samples are needed to estimate the sample variance even for projections shorter than 200 years.

The variability in the results presented here was only present due to the 
Table 4: Variance, skewness and kurtosis obtained with 25000 MC runs at different census epochs. "Mitigation only" scenario from Table 1.

\begin{tabular}{c||llll}
\multicolumn{1}{c||}{ Distribution parameter } & \multicolumn{4}{c}{ Census epoch } \\
& $\mathbf{2 0 6 3}$ & $\mathbf{2 1 1 3}$ & $\mathbf{2 1 6 3}$ & $\mathbf{2 2 1 3}$ \\
\hline \hline Variance (no. objects $^{2}$ ) & $5.87 \mathrm{E} 6$ & $8.05 \mathrm{E} 6$ & $1.08 \mathrm{E} 7$ & $1.14 \mathrm{E} 7$ \\
Skewness (unitless) $_{\text {Kurtosis (unitless) }}$ & 0.83 & 0.62 & 0.53 & 0.47 \\
Kurs & 1.05 & 0.55 & 0.33 & 0.31
\end{tabular}

differences in the number of collisions in different MC runs, as well as their epochs and resulting numbers of fragments. This is to say that the presented results follow a simplified scenario, which does not account for all the uncertainties affecting predictions of the future space debris population (DoladoPerez et al., 2015). That being said, the investigated scenario is the one on which the discussion regarding e.g. space debris remediation is based (Liou et al., 2013). Therefore, investigating the uncertainty in the results of this scenario is of particular importance.

\section{Conclusions}

It was shown that at most 60 Monte Carlo runs of space debris simulations are sufficient to estimate the mean number of objects in orbit with precision of $10 \%$. This is consistent with the findings of previous studies.

Previous space debris studies used means of the Monte Carlo samples to present the results, sometimes accompanied by standard deviation bands. It was shown here that the distribution of the number of objects in 2213 and earlier census epochs is most likely not normal. Therefore, means with standard deviations may not convey the information needed in decision making relating to the management of the debris environment, because they do not closely represent the underlying probability density function (Wisniewski, 2009). Associated with this nature of the distribution is the fact that it is more probable that the number of objects in orbit will be below rather than above the mean.

It was shown that Monte Carlo samples larger by orders of magnitude than traditionally used are needed to represent the uncertainty in the final distributions of the number of objects as well as the number of catastrophic collisions, even for projections shorter than 200 years. No definite minimum size of the sample can be recommended, however. Certain investigations, e.g. 


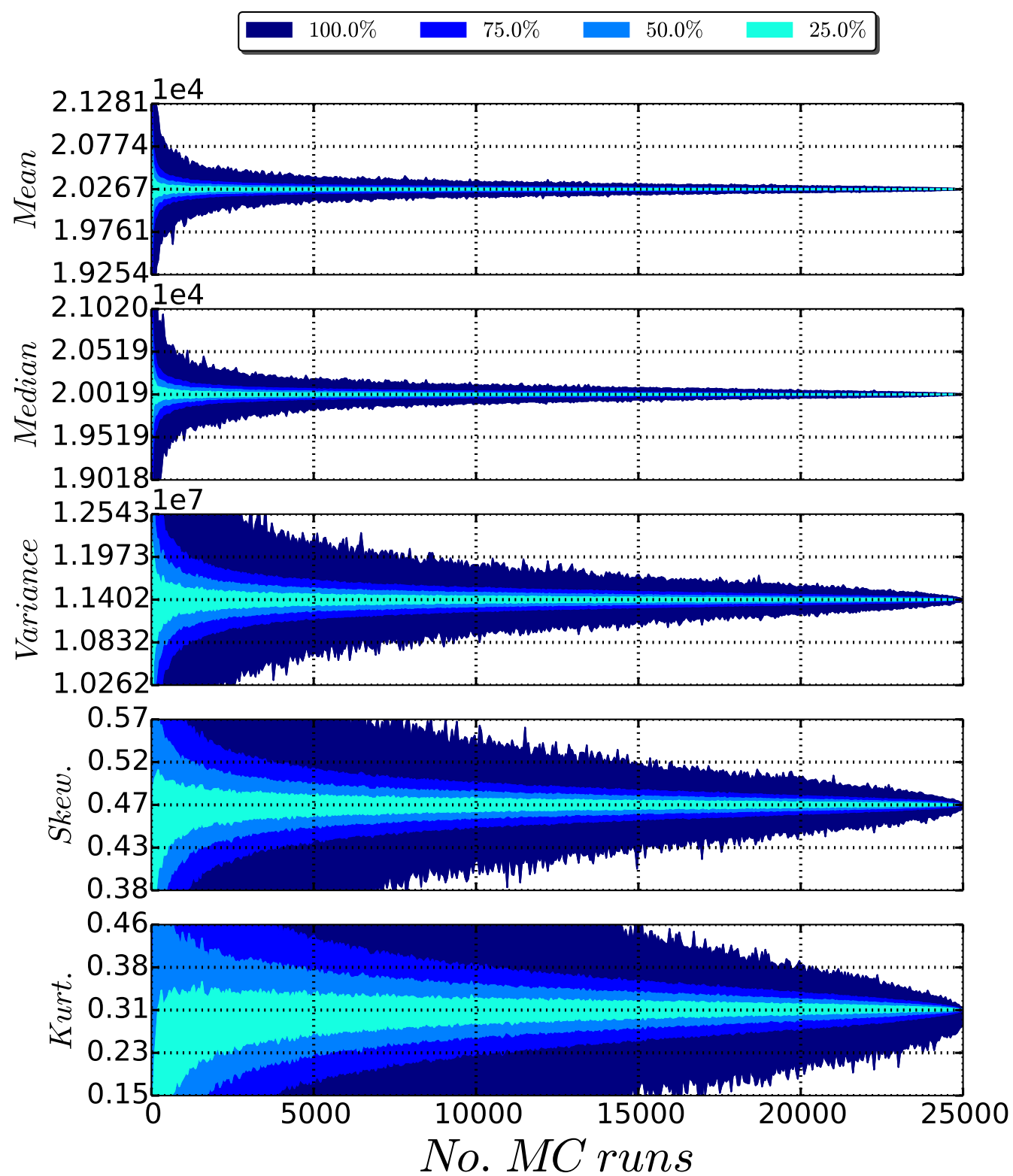

Figure 11: Variation of the mean, median, variance, skewness and kurtosis of the number of objects in 2213 with the sample size. "Mitigation only" scenario from Table 1, details given in text. 


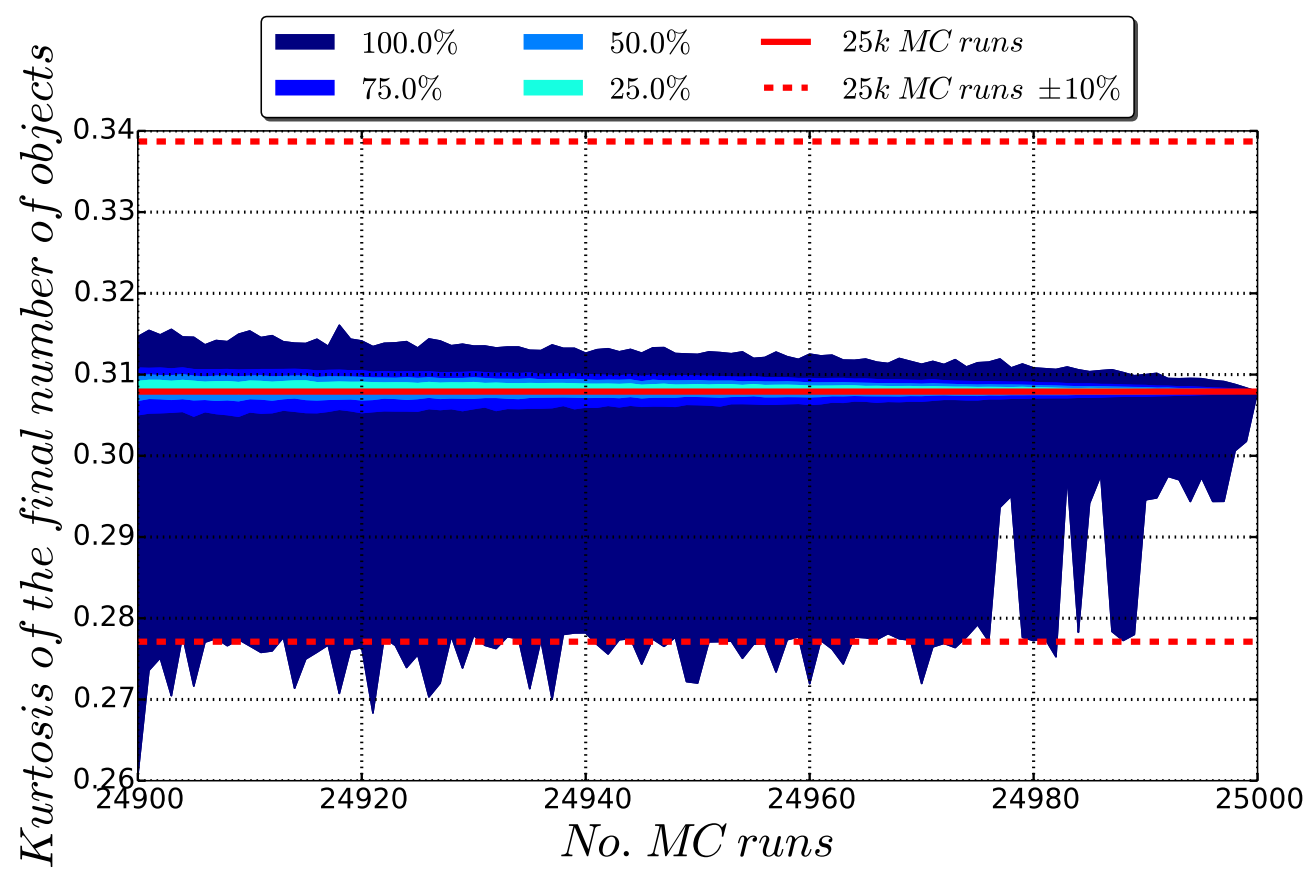

Figure 12: Variation of the kurtosis of the number of objects in the MC sample of the "mitigation only" scenario from Table 1 at the end of the projection (2213) with the sample size. Showing the largest sample sizes that were examined. Monte Carlo runs corresponding to the given sample size were randomly selected 2000 times, and the indicated percentiles of the distribution computed for every sample size. Also showing the value obtained with $25000 \mathrm{MC}$ runs, $\pm 10 \%$. 


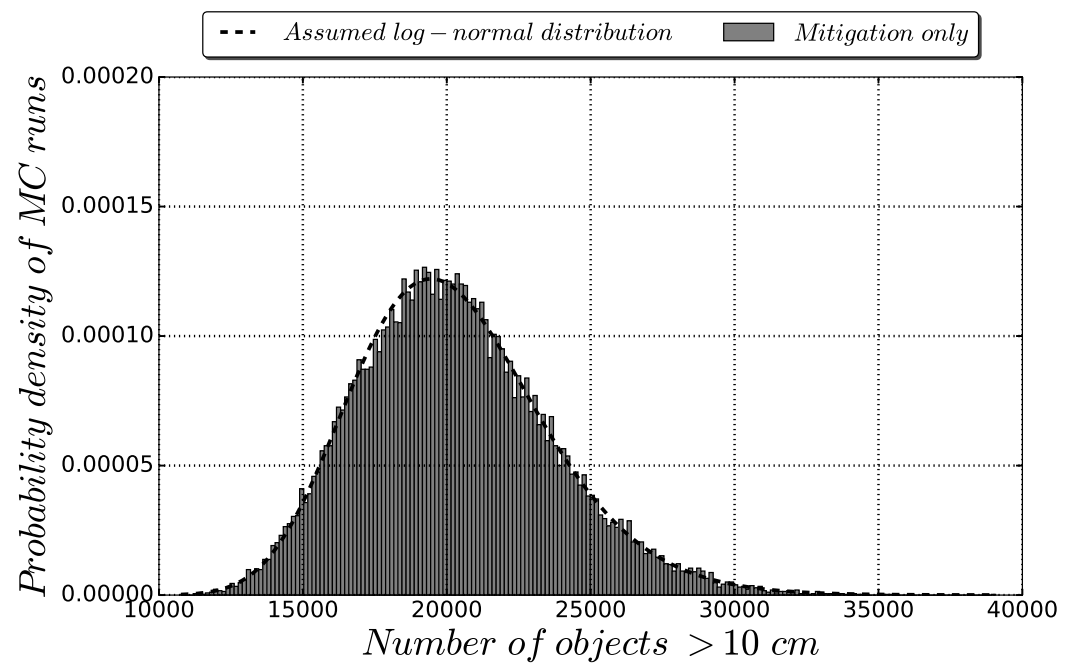

(a) 2213

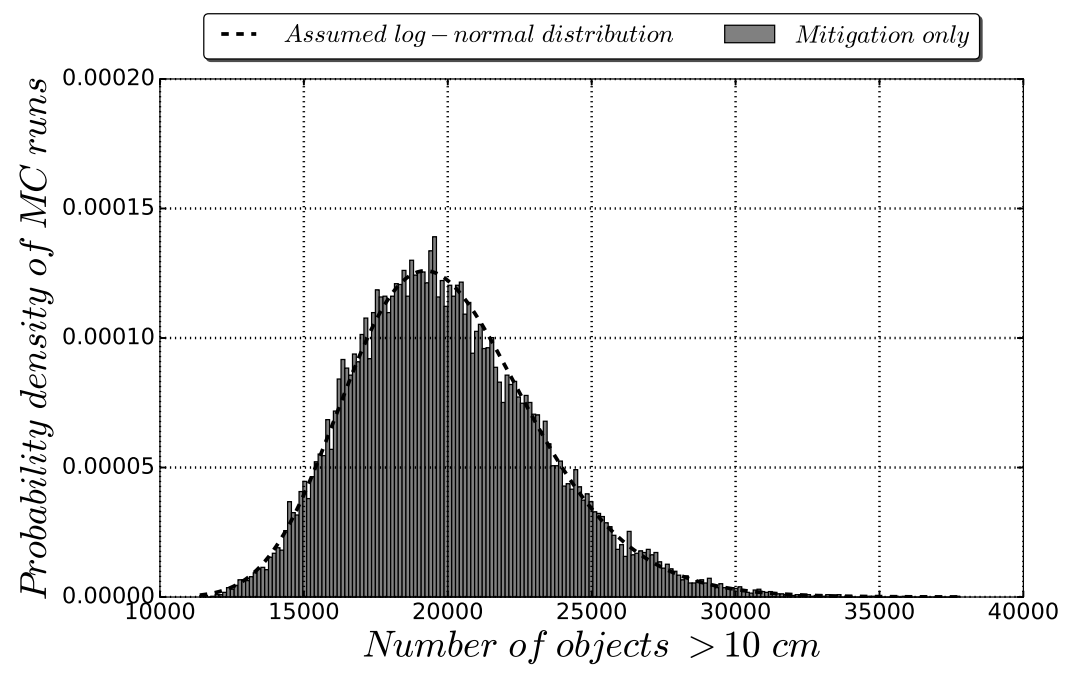

(b) 2163

Figure 13: Histograms comparing the Monte Carlo sample distributions of objects larger than $10 \mathrm{~cm}$ in 2213 and 2163 to the assumed theoretical lognormal distributions. "Mitigation only" scenario from Table 1 projected with 25000 Monte Carlo runs. 


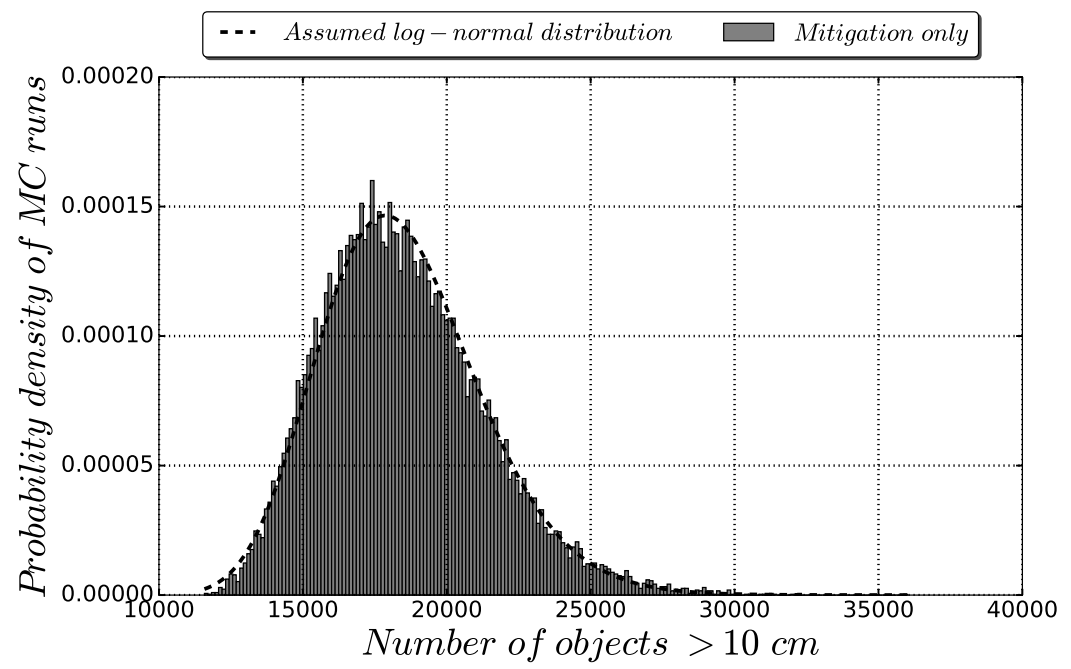

(a) 2113

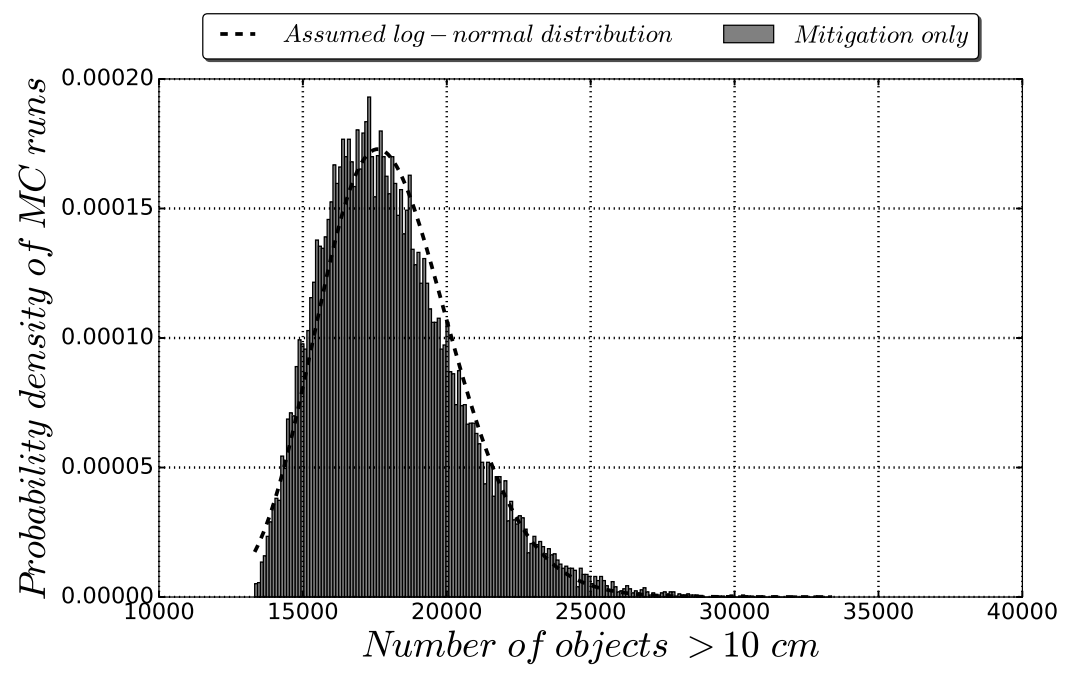

(b) 2063

Figure 14: Histograms comparing the Monte Carlo sample distributions of objects larger than $10 \mathrm{~cm}$ in 2113 and 2063 to the assumed theoretical lognormal distribution. "Mitigation only" scenario from Table 1 projected with 25000 Monte Carlo runs. 
comparing one mitigation strategy to another, may be carried out based on samples using only tens of MC runs. Others, bearing larger consequences, e.g. making decisions whether to implement active debris removal, require larger samples to ensure that the uncertainty in the results is well captured. It is recommended to carry out bootstrap analyses on higher orders of the distribution, not only on the mean as done thus far. One should also examine the probability density at the largest outliers in the sample and be satisfied that probability of generating even larger outliers is as close to zero as deemed appropriate for the analysis at hand.

It should be appreciated that active debris removal may be unnecessary to halt the increase of the number of objects in orbit. In order to make a decision about implementing ADR, the uncertainty in the outcome of performing or not performing ADR should be taken into account. In order to do this, ADR should be simulated such that the distribution of the number of objects in orbit is reliably captured, and compared to this (or a similar) study where ADR is not implemented.

If certainty about whether or not to perform any actions cannot be ascertained with traditionally used small MC samples, even less can presumably be inferred regarding the specific details of the policies or actions needed. For example, it is unlikely that decisions about how many objects should be removed from the environment, which ones and in what order, can be relied upon fully if they do not use Monte Carlo samples larger than were traditionally used.

Overall, it is recommended that the way in which space debris simulation results are presented is revised to incorporate the uncertainty information in an easy to understand manner, e.g. by quoting the probability that a given action will achieve a certain result (Wisniewski, 2009). This uncertainty should also be appreciated by policy makers and actors with authority to shape the future of the space debris environment. However, in order to have confidence in the uncertainty estimated using current evolutionary debris models, larger Monte Carlo samples are needed.

\section{Acknowledgements}

The authors acknowledge the use of the IRIDIS High Performance Computing Facility, and associated support services at the University of Southampton, in the completion of this work. 
The authors would like to thank the ESA Space Debris office for allowing the use of the MASTER 2013 reference population.

Aleksander Lidtke was funded by an EPSRC Doctoral Training Grant awarded by the Faculty of Engineering and the Environment of University of Southampton. The numbers of objects in orbit, recorded in 25000 Monte Carlo runs, as well as the corresponding epochs can be downloaded from the following link: http://dx.doi.org/10.5258/SOTON/397452.

\section{References}

Ariyoshi, Y., \& Hanada, T., GEODEEM 4.0: Updated Model for Better Understanding GEO Debris Environment, 27th International Symposium on Space Technology and Science, Tsukuba, Japan, 2009.

Bastida-Virgili, B., DELTA (Debris Evolution Long-Term Analysis), 6th International Workshop on Astrodynamics Tools and Techniques, Darmstadt, Germany, 2016.

Blake, R., \& Lewis, H.,G., The effect of modelling assumptions on predictions of the space debris environment, 65th International Astronautical Congress, Toronto, Canada, IAC-14.A6.2.2, 2014.

Braun, V., Luepken, A., Flegel, S., Gelhaus, J., Moeckel, M., Kebschull, C., Wiedemann, C., \& Voersmann, P., Active debris removal of multiple priority targets, Adv. Space Res., 51, 1638-1648, 2013.

DeGroot, M.H., \& Schervish, M.J., Probability and Statistics, Fourth Edition, Pearson Education Limited, Harlow, UK, 2014.

Dolado-Perez, J.C., Pardini, C., \& Anselmo, L., Review of uncertainty sources affecting the long-term predictions of space debris evolutionary models, Acta Astronautica, 113, 51-65, 2015.

Farinella, P., \& Cordelli, A., The Proliferation of Orbiting Fragments: a Simple Mathematical Model, Science and Global Security, 2, 365-378, 1991.

Furuta, S., Hanada, T., Fujita, K., \& Takezono, K., Discussion on the Necessity of Orbital Debris Removal in the Geostationary Region, 65th International Astronautical Congress, Toronto, Canada, IAC-14.A6.2.2, 2014. 
Johnson, N.L., Krisko, P.H., Liou, J.C., \& Anz-Meador, P.D., NASA's new breakup model of evolve 4.0, Adv. Space Res., 28, 1377-1384, 2001.

Johnson, N., The Historical Effectiveness of Space Debris Mitigation Measures, 56th International Astronautical Congress, Fukoka, Japan, IAC05.B6.3.07, 2012.

Kebschull, C., Krag, H., Braun, V., Hesselbach, S., Radtke, J., \& Scheidemann, P., Simulation of the space debris environment in LEO using an analytical approach, 40th COSPAR Scientific Assembly, Moscow, Russia, 2014.

Kessler, D.J., \& Cour-Palais, B.G., Collision frequency of artificial satellites: The creation of a debris belt, J. Geophys. Res., 83 (A6), 2637-2646, 1978.

Kessler, D., Derivation of the Collision Probability between Orbiting Objects: The Lifetimes of Jupiter's Outer Moons, Icarus, 48, 39-48, 1981.

Klinkrad, H., Space Debris: Models and Risk Analysis, Praxis Publishing, Ltd., Chichester, UK, 3-540-25448-X, 2006.

Lewis, H.G., Swinerd, G.G., Williams, N., \& Gittins, G., DAMAGE: a dedicated GEO debris model framework, 3rd European Conference on Space Debris, Noordwijk, Netherlands, 2001.

Lewis, H.G., Swinerd, G.G., Newland, R.J., \& Saunders, A., The fast debris evolution model, Adv. Space Res., 44, 568-578, 2009.

Lewis, H.G., White, A.E., \& Stokes, H., The effectiveness of space debris mitigation measures, ISU's 16th Annual International Symposium, 2012.

Lewis, H.G. \& Horbury, T., Implications of Prolonged Solar Minimum Conditions for the Space Debris Population, 6th European Conference on Space Debris, Darmstadt, Germnay.

Liou, J.C., Collision activities in the future orbital debris environment, Adv. Space Res., 38, 2102-2106, 2006.

Liou, J.C., Johnson, N.L., Risks in Space from Orbiting Debris, Science, 311, 340-341, 2006. 
Liou, J.C., \& Johnson, N.L., Instability of the present LEO satellite populations, Adv. Space Res., 41, 1046-1053, 2008.

Liou, J.C., A statistical analysis of the future debris environment, Acta Astronautica, 62, 264-271, 2008.

Liou, J.C., \& Johnson, N.L., A sensitivity study of the effectiveness of active debris removal in LEO, Acta Astronautica, 64, 236-243, 2009.

Liou, J.C., An active debris removal parametric study for LEO environment remediation, Adv. Space Res., 47, 1865-1876, 2011.

Liou, J.C., Anilkumar, A.K., Bastida Virgili, B., Hanada, T., Krag, H., Lewis, H.G., Raj, M.X.J., Rao, M.M., Rossi, A., \& Sharma, R.K., Stability of the Future LEO Environment - an IADC Comparison Study, 6th European Conference on Space Debris, Darmstadt, Germany, 2013.

McKnight, D., DiPentino, F., \& Knowles, S., Massive Collisions in LEO - a catalyst to initiate ADR, Proceedings of the 65th International Astronautical Congress, Toronto, Canada, IAC-14.A6.2.1, 2014.

Oepik, E.J., Collision probabilities with the planets and the distribution of interplanetary matter, Proceedings of the Royal Irish Academy. Section A: Mathematical and Physical Sciences, 54, 165-199, 1952.

Pas, N., Lousada, J., Terhes, C., Bernabeu, M., \& Bauer, W., Target selection and comparison of mission design for space debris removal by DLRs advanced study group, Acta Astronautica, 102, 241-248, 2014.

Radtke, J., Flegel, S., Gelhaus, J., Moeckel, M., Braun, V., Kebschull, C., Wiedemann, C., Krag, H., Merz, K., \& Voersmann, P., Revision of statistical collision analysis for objects inside of satellite constellations, Proceedings of the 64th International Astronautical Congress, Beijing, China, IAC-13,A6,P,8, 2013.

Radtke, R., \& Stoll, E., Comparing long-term projections of the space debris environment to real world data Looking back to 1990, Acta Astronautica, 127, 482-490, 2016.

The Scipy community, scipy.stats.describe, Last accessed: 21 September 2015, URL: www.docs.scipy.org/doc/scipy/reference/generated/ scipy.stats.describe.html, 2015. 
Space-Track, Last accessed: 10 March 2016, URL: www.space-track.org, 2016.

Talent, D.L., Analytic Model for Orbital Debris Environmental Management, The Journal of Spacecraft and Rockets, 29, 508-513, 1992.

Vallado, D., \& Seago, J.H., Covariance realism, AAS-AIAA Astrodynamics Specialist Conference, Pittsburgh, USA, 2009.

White, A.E., \& Lewis, H.G., The many futures of active debris removal, Acta Astronautica, 95, 189-197, 2014.

Wisniewski, M, Quantitative methods for decision makers : Fifth Edition, Financial Times Press, 2009.

Wormnes, K., Le Letty, R., Summerer, L., Schonenborg, R., Dubois-Matra, O., Luraschi,E., Cropp, A., Krag, H., Delaval, J., ESA technologies for space debris remediation, 6th European Conference on Space Debris, Darmstadt, Germany, 2013. 